\section{Vacunas anti-neumocóccicas en población pediátrica: actualización}

\section{Conjugate pneumococcal vaccines in pediatric population: up to date}

\section{Sr. Editor:}

Hemos leído con interés el artículo: "Vacunas antineumocóccicas en población pediátrica: actualización”, en el cual se hace una revisión sobre las vacunas antineumocóccicas y se discute el impacto en Chile de la vacuna antineumocóccica 10 -valente en la cual la mayoría de los serotipos está conjugada a proteína $\mathrm{D}$ de $\mathrm{H}$. influenzae (PCV10). Se concluye que sería un buen momento para considerar un cambio en la vacuna seleccionada para Chile, a favor de la vacuna antineumocóccica 13-valente (PCV13), basado en tres consideraciones: la "no evidencia" de efecto de rebaño, el incremento en la circulación del serotipo 3 (ST3) y una mayor cobertura teórica de serotipos (ST). No obstante, encontramos que no fueron considerados algunos de los datos y análisis más recientes de eficacia y efectividad de PCV10 y PCV13, los cuales creemos podrían reconsiderar dicha conclusión ${ }^{2-6}$.

Primero, el autor menciona que no hubo un efecto de rebaño en Chile, enfocándose únicamente en el "discreto aumento" en casos totales en el grupo de $>65$ años durante el último año, lo cual en teoría se debe a un leve incremento de ST vacunales (STV) y ST no vacunales (STNV). En realidad, en dicho rango de edad, se evidencia una reducción de $>50 \%$ en proporción de cada uno de los cuatro STV más importantes: 14, 1,7F, y $6 \mathrm{~B}^{7}$. Siendo que la presencia de un efecto de rebaño se define por una caída específica de casos causados por STV podemos concluir que ya existen señales de efecto de rebaño en la población no vacunada. Efectos similares fueron reportados en otros países que utilizan PCV10, así como un impacto en la portación nasofaríngea ${ }^{2,8,9}$. Debe sorprender que tal efecto es visto a pesar de una cobertura de sólo $68 \%$ para la dosis de refuerzo, y en la ausencia de la implementación de un programa de dosis de nivelación ${ }^{10}$.

Los otros argumentos del autor, a favor de considerar el cambio de la vacuna, se basan esencialmente en que PCV 13 contiene tres serotipos adicionales: 6A, 19A, y 3. Tras la introducción de PCV10, los casos por STV en niños bajo los dos años de edad disminuyeron en un $88,2 \%$; también se ha observado una notable reducción en los ST relacionados 6A $(87,9 \%)$ y 19A (36\%). Este hallazgo, que sugiere protección cruzada por PCV10, también ha sido reportado en otros lugares ${ }^{6}$. Con respecto al "incremento" en ST3, trabajos previos en Chile y otros países ya han reportado una variabilidad natural en su prevalencia (coeficiente de variación en Chile 81,7\%) ) $^{7,11}$. $\mathrm{La}$ apertura del nicho creado por la vacunación podría amplificar estas tendencias seculares ${ }^{12}$. Por otra parte, los estudios clínicos y post-introducción de PCV13 no ofrecen evidencia robusta de protección contra el mismo probablemente debido a que el ST3 posee una cápsula distinta y densa la cual le otorga cierta capacidad para evadir la respuesta inmune 6 .

Por lo arriba expresado consideramos que existen señales de protección de rebaño en Chile; que el incremento aparente en circulación de ST3 observado en adultos no es necesariamente relacionado con el uso de la vacuna en niños; y que la estimación de la potencial cobertura vacunal debe tomar en cuenta la protección cruzada probable de PCV10 y las dudas en cuanto al efecto de PCV13 sobre el ST3. Finalmente, más allá del número de serotipos incluidos en una vacuna, su beneficio debe ser evaluado sobre la carga total de la enfermedad neumocóccica que previene (enfermedad invasora, neumonía, y otitis media aguda), para lo cual existe evidencia robusta en el caso de PCV10 $0^{2-6}$.

\section{Referencias bibliográficas}

1.- Potin M. Vacunas anti-neumocóccicas en población pediátrica: actualización. Rev Chilena Infectol 2014; 31 : 452-6.

2.- Palmu A A, Jokinen J, Borys D, Nieminen H, Ruokokoski E, Siira L, et al. Effectiveness of the ten-valent pneumococcal Haemophilus influenzae protein D conjugate vaccine (PHiD-CV10) against invasive pneumococcal disease: a cluster randomised trial. Lancet 2013; 381: 214-22.

3.- Palmu A A, Jokinen J, Nieminen H, Syrjanen R, Ruokokoski E, Puumalainen T, et al. Vaccine effectiveness of the pneumococcal Haemophilus influenzae protein D conjugate vaccine (PHiD-CV10) against clinically suspected invasive pneumococcal disease: a cluster-randomised trial. Lancet Respir Med 2014; 2: 717-27.

4.- Domingues C M, Verani J R, Montenegro Renoiner E I, de Cunto Brandileone M C, Flannery B, de Oliveira L H, et al. Effectiveness of ten-valent pneumococcal conjugate vaccine against invasive pneumococcal disease in Brazil: a matched case-control study. Lancet Respir Med 2014; 2 : 464-71.

5.- Tregnaghi M W, Saez-Llorens X, Lopez P, Abate H, Smith E, Posleman A, et al. Efficacy of pneumococcal nontypable Haemophilus influenzae protein D conjugate vaccine (PHiD-CV) in young Latin American children: A double-blind randomized controlled trial. PLoS medicine 2014; 11 (6): e1001657.

6.- Hausdorff W P, Hoet B, Adegbola R A. Predicting the impact of new pneumococcal conjugate vaccines: serotype composition is not enough. Expert Rev Vaccines 2014: 1-16.

7.- Boletín Instituto de Salud Pública de Chile. Vigilancia de enfermedad invasora Streptococcus pneumoniae. Chile, 2007-2013. Marzo 2014; 4 (3):1-24. http://www.ispch.cl/ sites/default/files/Boletin_Neumo_21-03-2014.pdf

8.- Palmu A A J J, Ruokokoski E, et al. Indirect impact of pneumococcal Haemophilus influenzae protein-D conjugate vaccine (PHID-CV10) on suspected nonconfirmed invasive pneumococcal disease (IPD) in cluster-randomized trial International Symposium on 


\section{Carta al Editor}

Pneumococci and Pneumococcal Diseases; Hyderabad, India 2014. http://registration-isppd14.kenes.com/ ISPPD14/CM.NET.WebUI/CM.NET.WEBUI.scpr/ SCPRfunctiondetail.aspx? confID $=05000000-0000$ 0000-0000-000000000043\&sesID $=05000000$ 0000-0000-0000-000000007808\&abs ID $=07000000-0000-0000-0000-000000031583$, Acceso: octubre de 2014

9.- Mrkvan T H W, Moreira M, et al. Evidence of early herd protection after infant vaccination with the 10-valent pneumococcal non-typeable Haemophilus influenzae protein D conjugate vaccine (PHiD-CV): a review. Nordic Vaccine Meeting; Bergen, Norway 2014. http://www.fhi.no/ dokumenter/d858c4c99b.pdf

10.- González C. Coberturas de vacunas: desafíos para el control de enfermedades inmunoprevenibles http://www. sochinf.cl/sitio/templates/sochinf2008/documentos/2014/ pre_vacunas/5_Dra_Gonzalez.pdf2014. Acceso: octubre de 2014.
11. Lagos R, Muñoz A, San Martín O, Maldonado A, Hormazabal J C, Blackwelder W C, et al. Age- and serotypespecific pediatric invasive pneumococcal disease: insights from systematic surveillance in Santiago, Chile, 1994-2007. J Infect Dis 2008;198: 1809-17.

12. Feikin D R, Kagucia E W, Loo J D, Link-Gelles R, Puhan M A, Cherian T, et al. Serotype-specific changes in invasive pneumococcal disease after pneumococcal conjugate vaccine introduction: a pooled analysis of multiple surveillance sites. PLoS Med 2013; 10: e1001517.

William Hausdorff, Alejandro Lepetic y Rodrigo De Antonio GlaxoSmithKline-Vacunas

Correspondencia a: William Hausdorff william.p.hausdorff@gsk.com 